\title{
Effect of ketamine pretreatment for anaesthesia in patients undergoing percutaneous transluminal balloon angioplasty with continuous remifentanil infusion
}

\author{
Na Hyung Jun ${ }^{1}$, Jae Kwang Shim ${ }^{1,2}$, Yong Sun Choi ${ }^{1,2}$, Seung Ho An ${ }^{1}$, and Young Lan Kwak ${ }^{1,2,3}$ \\ ${ }^{1}$ Department of Anaesthesiology and Pain Medicine, ${ }^{2}$ Anaesthesia and Pain Research Institute, Yonsei University College of Medicine, \\ ${ }^{3}$ Severance Biomedical Science Institute, Seoul, Korea
}

Background: An appropriate level of sedation and pharmacological assist are essential during percutaneous transluminal balloon angioplasty (PTA). Ketamine provides good analgesia while preserving airway patency, ventilation, and cardiovascular stability with an opioid sparing effect suggesting that it would be ideal in combination with remifentanil and midazolam in spontaneously breathing patients. We evaluated the effect of a small dose of ketamine added to midazolam and remifentanil on analgesia/sedation for PTA procedures.

Methods: Sixty-four patients receiving PTA were enrolled. The Control group received midazolam $1.0 \mathrm{mg}$ i.v. and continuous infusion of remifentanil $0.05 \mu \mathrm{g} / \mathrm{kg} / \mathrm{min}$. The Ketamine group received, in addition, an intravenous bolus of $0.5 \mathrm{mg} / \mathrm{kg}$ ketamine. Patients' haemodynamic data were monitored before remifentanil infusion, 5 min after remifentanil infusion, at 1, 3, 5, 30 min after incision, and at admission to the recovery room. Verbal numerical rating scales (VNRS) and sedation [OAA/S (Observer's Assessment of Alertness/Sedation)] scores were also recorded.

Results: The VNRS values at 1, 3, and $5 \mathrm{~min}$ after incision and OAA/S scores at $5 \mathrm{~min}$ after remifentanil infusion, and 1, 3, and 5 min after incision were lower in the Ketamine group than in the Control group. In the Control group, the VNRS value at $1 \mathrm{~min}$ after incision significantly increased and OAA/S values at 3, 5, and $30 \mathrm{~min}$ after incision significantly decreased compared to baseline values, while there were no significant changes in the ketamine group.

Conclusions: A small dose of ketamine as an adjunct sedative to the combination of midazolam and remifentanil produced a better quality of sedation and analgesia than without ketamine and provided stable respiration without cardiopulmonary deterioration. (Korean J Anesthesiol 2011; 61: 308-314)

Key Words: Ketamine, Pain scale, Remifentanil, Sedation.

Received: February 1, 2011. Revised: 1st, March 11, 2011; 2nd, March 22, 2011. Accepted: April 6, 2011.

Corresponding author: Young Lan Kwak, M.D., Ph.D., Department of Anaesthesiology and Pain Medicine, Yonsei University College of Medicine, 134, Shinchon-dong, Seodaemun-gu, Seoul 120-752, Korea. Tel: 82-2-2228-8513, Fax: 82-2-364-2951, E-mail: ylkwak@yuhs.ac (c) This is an open-access article distributed under the terms of the Creative Commons Attribution Non-Commercial License (http:// creativecommons.org/licenses/by-nc/3.0/), which permits unrestricted non-commercial use, distribution, and reproduction in any medium, provided the original work is properly cited. 


\section{Introduction}

For successful percutaneous transluminal balloon angioplasty (PTA), sedation is essential so that patients remain immobile during the procedure in an uncomfortable environment. Since it takes a long time and is sensitive to motion artifacts [1], moderate sedation/analgesia and depressing consciousness that is, patients responding purposefully to verbal commands either alone or accompanied by light tactile stimulation with spontaneous ventilation, are usually required during PTA procedures [2].

Midazolam and various opioids are frequently used in combination to provide sedation, anxiolysis and analgesia during short-term surgical procedures or interventional radiologic procedures [3]. Remifentanil has become popular lately as one of the intravenous opioids, because it can be infused continuously during simple procedures, regarding its excellent analgesic effect, and has a rapid onset and offset of action owing to a short context sensitive half-time $[4,5]$. Furthermore, as shown in a review reporting the incidence of serious cardiopulmonary complications and death as 5.4 and 0.3 per 1,000 , respectively in patients who underwent sedation with benzodiazepine and supplemental narcotics, the combination of both drugs could result in cardiopulmonary compromise [6]. To reduce the complications of intravenous anaesthetics, it is recommended that one considers combinations of various agents with different action mechanisms and at lower doses [7].

Ketamine in subhypnotic doses provides good analgesia while preserving airway patency, ventilation, and cardiovascular stability [8]. A small dose of ketamine may increase the analgesic potency of an opiate [9]. However, only a few studies have demonstrated the effect of ketamine on moderate sedation using a benzodiazepine and an opioid agent.

This study tested the hypothesis that adding a small dose of ketamine to midazolam and remifentanil combination can provide more effective analgesia/sedation for PTA procedures without adverse effects compared with a combination of midazolam and remifentanil only.

\section{Materials and Methods}

From 2008 to 2010, we prospectively studied 64 patients aged 27 to 84 years who underwent PTA. The study was approved by the hospital ethics committee, and written informed consent was obtained from all participants. All patients had American Society of Anesthesiologists (ASA) physical status I to III. Patients were excluded if they had ASA status IV, hepatic or renal insufficiency, history of allergy to the drugs used, need for inotropic agents, or need for mechanical ventilation. Patients were randomly assigned to one of the two treatment groups.
During the entire study, an anesthesiologist who was blinded to patient group assignment recorded the variables.

No premedication was given to the patients. The Control group received normal saline, the same amount of $0.5 \mathrm{mg} / \mathrm{kg}$ of ketamine and then were managed with the standard protocol including intravenous injection of $1.0 \mathrm{mg}$ of midazolam and continuous infusion of remifentanil $0.05 \mu \mathrm{g} / \mathrm{kg} / \mathrm{min}$. The Ketamine group received the sedation protocol consisting of an intravenous bolus of $0.5 \mathrm{mg} / \mathrm{kg}$ ketamine followed by a bolus of $1.0 \mathrm{mg}$ midazolam and continuous infusion of remifentanil $0.05 \mu \mathrm{g} / \mathrm{kg} / \mathrm{min}$. If signs of insufficient analgesia (facial grimace, movement, sudden increase in heart rate, spontaneous complaint of pain) were observed, an additional intravenous bolus of remifentanil $0.2 \mu \mathrm{g} / \mathrm{kg}$ was injected without changing the infusion rate of remifentanil in both groups. A local anesthetic agent was injected into the incision site before the incision by a cardiologist. Oxygen was delivered by a face mask to all patients throughout the procedure. Any episodes of hypoventilation (spontaneous $\mathrm{RR}<8 / \mathrm{min}$ ), desaturation $\left(\mathrm{SpO}_{2}\right.$ $<90 \%$ ) or apnea during the infusion phase was recorded and the jaw thrust maneuver was applied to patients, if necessary. When those episodes persisted even with stimulation, the remifentanil infusion rate was reduced to $0.03 \mu \mathrm{g} / \mathrm{kg} / \mathrm{min}$.

The patients were monitored for mean arterial blood pressure (MAP), heart rate (HR), peripheral oxygen saturation $\left(\mathrm{SpO}_{2}\right)$, and respiratory rate (RR) before the remifentanil infusion, $5 \mathrm{~min}$ after remifentanil infusion (baseline value), at $1,3,5,30 \mathrm{~min}$ after incision, and at admission to the recovery room. The verbal numerical rating scales (VNRS), sedation score [OAA/S (Observer's Assessment of Alertness/Sedation)] and the presence of nausea, vomiting, itching sense or apnea were recorded at the same time points. The quality of analgesia was assessed by VNRS using and 11 point scale (0-10) [9]. The patient was asked to rate the intensity of the pain experienced on the scale of with 0 being "no pain" and 10 "the worst pain imaginable". Such scales have the advantages of being quick and simple, require no equipment, and they are easy to understand [10]. The OAA/S scale was developed to measure the level of alertness in subjects who are sedated and is defined in Appendix A. The duration of remifentanil infusion, total amount infused, and the frequency of bolus injection were recorded and the degree of satisfaction (1-4) of the surgeon and the patients were evaluated. Time to Aldrete score $>9$ was also recorded at the recovery room. The Aldrete score is a measure of recovery after anesthesia that includes level of consciousness, physical activity, hemodynamic stability, respiratory stability, and oxygen saturation status; scores for each category were 0 to 2 .

Statistical analysis was performed using SPSS 13.0 (SPSS Inc., Chicago, IL, USA). The normality of distribution was assessed with a q-q plot and the Shapiro-Wilk test. Patient characteristics 
(age, height, weight, duration of remifentanil infusion) were analysed using an independent t-test. Categorical variables (remifentanil bolus number, apnea after bolus, amnesia at recovery room and side effects) were evaluated by a $\chi^{2}$ test or a Fisher's exact test when appropriate. Repeated measures analysis of variance (ANOVA) was used for the comparison of and VNRS scores for pain. If significant differences were detected, differences between two means were compared by an independent t-test with Bonferroni correction. Comparisons between the two groups with regard to pain and sedation scores were done with the Mann-Whitney U test, and intragroup comparisons were done with the Wilcoxon signed rank test. All values were expressed as mean \pm standard deviation, median (interquartile range) or the number of patients. A P value $<0.05$ was considered statistically significant. Sample size calculations were based on previous studies. To detect a two-point difference in VNRS score for pain (two-sided $\alpha$ of $5 \%$ and $\beta$ of $20 \%$ ), 32 subjects were required in each group.

\section{Results}

Demographic and clinical parameters were comparable between the groups. The duration of remifentanil infusion (80 \pm $39 \mathrm{~min}$ vs. $71 \pm 28 \mathrm{~min}$ ) and the total amount infused (278 \pm 164 $\mu \mathrm{g}$ vs. $246 \pm 176 \mu \mathrm{g}$ ), apnea after bolus ( 4 cases vs. 3 cases) were higher in the Control group but none of these differences were statistically significant (Table 1).

The satisfaction scale ( 1 to 4 ) of the surgeons and the patients were high in both groups (4 vs. 4). Between group differences in haemodynamic and respiratory parameters during and after the procedures were not significant except for respiratory rates. After skin incision, respiratory rates were significantly lower in

Table 1. Patient Demographics and Preoperative Clinical Data

\begin{tabular}{|c|c|c|c|}
\hline & $\begin{array}{l}\text { Control group } \\
\quad(\mathrm{n}=32)\end{array}$ & $\begin{array}{l}\text { Ketamine group } \\
\quad(\mathrm{n}=32)\end{array}$ & P value \\
\hline Age (years) & $67 \pm 11$ & $61 \pm 11$ & 0.055 \\
\hline Female gender, \# (\%) & $11(34.4)$ & $8(25.0)$ & 0.412 \\
\hline ASA I & $3(9.4)$ & $3(9.4)$ & 0.601 \\
\hline ASA II & $29(90.6)$ & $28(87.5)$ & \\
\hline ASA III & 0 & $1(3.1)$ & \\
\hline Height $(\mathrm{cm})$ & $163.2 \pm 7.9$ & $163.2 \pm 8.2$ & 0.983 \\
\hline Weight (kg) & $63.4 \pm 12.1$ & $63.4 \pm 8.8$ & 0.945 \\
\hline Duration of procedure (min) & $82 \pm 29$ & $76 \pm 30$ & 0.488 \\
\hline Remifentanil infusion duration (min) & $80 \pm 39$ & $70 \pm 28$ & 0.211 \\
\hline Total amount of remifentanil infused ( $\mu \mathrm{g})$ & $278 \pm 164$ & $264 \pm 157$ & 0.729 \\
\hline Number of remifentanil bolus injections & $1.3 \pm 1.3$ & $1.0 \pm 1.5$ & 0.479 \\
\hline Apnea after remifentanil bolus injection & $4(12.5)$ & $3(9.4)$ & 0.689 \\
\hline Amnesia at the recovery room & $12(37.5)$ & $16(50.0)$ & 0.313 \\
\hline Patient satisfaction $(1-4)$ & $4(3-4)$ & $4(3-4)$ & 0.843 \\
\hline Surgeon satisfaction $(1-4)$ & $4(3-4)$ & $3.5(3-4)$ & 0.319 \\
\hline Time to Aldrete score $>9$ (min)* & $5.1 \pm 5.3$ & $3.8 \pm 4.3$ & 0.287 \\
\hline
\end{tabular}

Data are expressed as mean \pm SD or number of patients (\%). ASA: American Society of Anesthesiologists. $*$ Measured after recovery room admission.

Table 2. Haemodynamic Data

\begin{tabular}{|c|c|c|c|c|c|c|c|c|}
\hline & Group & T0 & $\mathrm{T} 1$ & $\mathrm{~T} 2$ & T3 & $\mathrm{T} 4$ & $\mathrm{~T} 5$ & $\mathrm{~T} 6$ \\
\hline \multirow[t]{2}{*}{$\mathrm{RR}$} & Control & $20 \pm 2^{\dagger}$ & $18 \pm 3$ & $20 \pm 4^{\dagger}$ & $23 \pm 4^{\dagger}$ & $20 \pm 4^{\dagger}$ & $21 \pm 6^{\dagger}$ & $19 \pm 3$ \\
\hline & Ketamine & $21 \pm 2^{\dagger}$ & $16 \pm 3$ & $16 \pm 3^{*}$ & $16 \pm 5^{*}$ & $15 \pm 4^{*}$ & $15 \pm 4^{*}$ & $16 \pm 3^{*}$ \\
\hline \multirow[t]{2}{*}{ MAP } & Control & $100 \pm 21$ & $96 \pm 17$ & $100 \pm 20$ & $94 \pm 17$ & $90 \pm 16$ & $89 \pm 15$ & $90 \pm 15$ \\
\hline & Ketamine & $98 \pm 17$ & $99 \pm 15$ & $95 \pm 14$ & $94 \pm 15$ & $91 \pm 16$ & $96 \pm 15$ & $97 \pm 14$ \\
\hline \multirow[t]{2}{*}{ HR } & Control & $73 \pm 17$ & $70 \pm 17$ & $70 \pm 17$ & $72 \pm 16$ & $70 \pm 16$ & $71 \pm 16$ & $73 \pm 16$ \\
\hline & Ketamine & $70 \pm 12$ & $71 \pm 12$ & $69 \pm 13$ & $68 \pm 13$ & $69 \pm 12$ & $69 \pm 12$ & $70 \pm 11$ \\
\hline \multirow[t]{2}{*}{$\mathrm{SpO}_{2}$} & Control & $99 \pm 2$ & $99 \pm 1$ & $99 \pm 2$ & $99 \pm 2$ & $99 \pm 2$ & $99 \pm 2$ & $99 \pm 1$ \\
\hline & Ketamine & $98 \pm 1$ & $99 \pm 1$ & $99 \pm 2$ & $99 \pm 2$ & $97 \pm 10$ & $99 \pm 2$ & $99 \pm 2$ \\
\hline
\end{tabular}

Data are expressed as mean \pm SD. T0: before remifentanil infusion, T1: 5 min after remifentanil infusion, T2: 1 min after incision, T3: 3 min after incision, T4: $5 \mathrm{~min}$ after incision, T5: $30 \mathrm{~min}$ after incision, T6: admission to recovery room, RR: respiratory rate, MAP: mean arterial pressure, HR: heart rate, $\mathrm{SpO}_{2}$ : peripheral oxygen saturation. ${ }^{*} \mathrm{P}<0.05$ compared with group 1 at each time point of measurement. ${ }^{\dagger} \mathrm{P}<0.01$ compared with T1. 
Table 3. Pain and Sedation Score

\begin{tabular}{llllrrrr}
\hline & \multicolumn{1}{c}{ Group } & \multicolumn{1}{c}{ T1 } & \multicolumn{1}{c}{ T2 } & \multicolumn{1}{c}{ T3 } & T4 & T5 \\
\hline \multirow{2}{*}{ VNRS } & Control & $0(0,1.75)$ & $2(1.25,5)^{\dagger}$ & $1.5(0,2.75)$ & $1.5(0,3)$ & $0(0,1)$ & $0(0,1)$ \\
& Ketamine & $0(0,0)$ & $0(0,1)^{*}$ & $0(0,1)^{*}$ & $0(0,0)^{*}$ & $0(0,2)$ & $0(0,1.75)$ \\
OAA/S & Control & $4(4,4)$ & $4(3,4)$ & $3(3,4)^{\dagger}$ & $3(3,4)^{\dagger}$ & $3(3,4)^{\dagger}$ & $4(3.25,4)$ \\
& Ketamine & $3(0.25,4)^{*}$ & $3(1,4)^{*}$ & $2.5(1,4)^{*}$ & $2(1,4)^{*}$ & $3(1.25,4)$ & $4(1.25,4)$ \\
\hline
\end{tabular}

Data are expressed as median (interquartile range). T1: 5 min after remifentanil infusion, T2: 1 min after incision, T3: 3 min after incision, T4: 5 min after incision, T5: 30 min after incision, T6: admission to recovery room, VNRS: verbal numerical rating scales, OAA/S: Observer's Assessment of Alertness/Sedation Scale. ${ }^{*} \mathrm{P}<0.05$ compared with group 1 at each time point. ${ }^{\dagger} \mathrm{P}<0.01$ compared with $\mathrm{T} 1$.

Table 4. Adverse Effects in the Two Groups

\begin{tabular}{lcc}
\hline & $\begin{array}{c}\text { Control group } \\
(\mathrm{n}=32)\end{array}$ & $\begin{array}{c}\text { Ketamine group } \\
(\mathrm{n}=32)\end{array}$ \\
\hline Apnea & 1 & 2 \\
Nausea & 1 & 0 \\
Vomiting & 0 & 0 \\
Itching & 2 & 0 \\
\hline
\end{tabular}

Data are expressed as number of patients.

the Ketamine group compared to the Control group (Table 2). Also, in the Control group, the respiratory rates significantly increased at 1, 3, 5, and 30 min after incision compared with the point when remifentanil was initially infused. In both groups, the respiratory rates significantly decreased $5 \mathrm{~min}$ after remifentanil infusion compare to rates before infusion.

VNRS values at 1, 3, and 5 min after incision were significantly lower in the Ketamine group than in the Control group. In the Ketamine group, lower OAA/S scores were found at $5 \mathrm{~min}$ after remifentanil infusion, and 1, 3, and 5 min after incision than in the Control group. In the Control group, VNRS value at $1 \mathrm{~min}$ after incision significantly increased and OAA/S values at 3,5 , and 30 min after incision significantly decreased compared to baseline values, while there were no significant changes in VNRS and OAA/S values in the Ketamine group (Table 3).

Apnea without a bolus developed in one patient in the Control group and two patients in the Ketamine group and all patients recovered self respiration after tactile stimulation without significant desaturation. Remaining side effects during the study were comparable between the groups and were not significant in both groups (Table 4).

\section{Discussion}

In this prospective study evaluating the effects of a small dose of ketamine as an adjunct sedative to the combination of midazolam and remifentanil in patients undergoing PTA, we found that the addition of a low dose of ketamine produced a better quality of sedation and analgesia than without ketamine, although the patients' satisfaction scores were high enough even in the Control group. The addition of ketamine provided more stable respiration without cardiopulmonary deterioration.

Sedation with various degrees of depth and duration is essential for both the patients' and interventionists' conveniences for interventional procedures. Medications should be administered to achieve: 1 . adequate analgesia, sedation, anxiolysis, and amnesia during the procedures, 2. control of unwanted motor behavior that inhibits the performance of procedures, 3. rapid return of the patient to consciousness, and 4. less risk of adverse events. For stable conditions of sedation and haemodynamics, continuous intravenous infusion rather than repetitive bolus doses [10] and combination of various agents having different action mechanisms are recommended. Therefore, combined use of analgesics and hypnotics, with some of them being short acting agents that permit continuous infusion, has been considered as a reasonable and safe sedation regimen for interventional procedures.

Midazolam, a short acting benzodiazepine, has been the most commonly used sedative agent for PTA. Midazolam produces dose-related sedation, amnesia, and anxiolysis, but has a poor analgesic effect, and recovery may be prolonged due to its cumulative hypnotic effect. Therefore, midazolam usually requires the use of an adjunctive analgesic agent. The synergistic effect of opioids and midazolam in combination is well known [11-14] and currently an alternative sedation protocol based on remifentanil with midazolam is widely used. Remifentanil is an ultra-short acting opioid popular in anaesthetic practices that is a mu-receptor agonist. The unique pharmacology of remifentanil, its rapid offset in particular, has attracted clinicians and investigators recently for procedures requiring a brief and intense opioid effect. The rapid offset of remifentanil, demonstrated by its extremely short context sensitive half-time (3-5 min), and which occurs regardless of the duration of the infusion, also allows continuous infusion for long procedures [15]. However, as for other opioids, remifentanil increases the risk of repetitive apnea and may produce dose dependent respiratory depression with delayed awakening. The most common side effects of combined use of midazolam and remifentanil are respiratory depression and patients' intolerance as a result of prolonged procedures [3]. 
Ketamine is a nonspecific N-methyl-d-aspartate (NMDA) receptor antagonist that provides intrinsic analgesia and apparent anesthesia with relative hemodynamic stability and protects airway reflexes $[16,17]$. Furthermore, a small-dose of ketamine increases thalamic sensory output and arousal [18]. Therefore, the administration of intravenous ketamine appears to be a safe and useful technique for monitored anesthesia care in the ambulatory setting. Ketamine has an additive hypnotic effect when used with midazolam [19]. In addition, a small dose of ketamine was reported to reduce postoperative hyperalgesia and opioid induced intolerance, especially tolerance to remifentanil. Both additive and beneficial effects of ketamine on midazolam and/or remifentanil effects would exert a beneficial effect on cardiopulmonary stability by decreasing the needed dose of both drugs. Studies on the combined use of ketamine and remifentanil are now being actively performed, but are mostly limited to patients undergoing general anesthesia [20,21].

In this study, we added a small dose of ketamine to a combination of midazolam and remifentanil for more satisfactory sedation with limited effects on cardio-respiratory function in patients undergoing PTA. We observed that the VNRS and OAA/S scores were significantly lower in the Ketamine group, demonstrating a better quality of sedation. Among various interventional procedures, PTA requires a long duration and motionless field. Therefore, deep sedation with spontaneous ventilation is needed during PTA. Frequently, a large amount of repetitive midazolam or a higher dose of remifentanil infusion are required, which may result in delayed awakening or significant respiratory depression, especially in compromised patients. Therefore, this study demonstrated that a small dose of ketamine given as a pre-procedural bolus intensified the analgesic and sedative effects of the drug combination without cardiorespiratory depression could have a significant clinical impact on PTA procedures. Most patients in the Ketamine group were in a painless and motionless state during the procedures, and their haemodynamics and respiration remained stable without adverse events. Interestingly, although ketamine was known to counteract opioid induced respiratory depression [22], the respiratory rates were significantly lower in the Ketamine group compared to the Control group. In both groups, the respiratory rates significantly decreased after remifentanil infusion (before the skin incision). Only in the Control group was the respiratory rate increased after incision, and it was higher than that in a previous study using midazolam and remifentanil [23]. This proves the superior sedative effect of the Ketamine group with regards to sedative quality. Although statistically not significant, heart rates were slightly lower throughout the procedure in the Ketamine group, which is opposite to its known characteristic of increasing heart rate. This may also have resulted from the effective sedation of ketamine pretreatment. There were no patients whose arterial oxygen saturation decreased below $90 \%$ in either group. Three patients developed apnea. All patients recovered self respiration immediately after verbal or gentle tactile stimulation without significant desaturation. The dose of remifentanil used in this study was significantly lower than in other studies of conscious analgesia and sedation $[23,24]$ and, therefore, opioid induced respiratory depression did not occur in our study. This was also associated with the fact that the expected opioid sparing effect of ketamine was not observed in this study. Based on the low VNRS and OAA/S scores as well as high satisfaction scores of both patients and care providers for both groups, we believe adequate sedation and analgesia were provided during the procedures, even in the Control group. The difference in remifentanil dosage from other studies might be related to racial differences. Significantly lower OAA/S value at $5 \mathrm{~min}$ after remifentanil infusion shows that ketamine may rapidly intensify the depth of sedation. Accordingly, in the Ketamine group, the VNRS score did not increase even after the skin incision, unlike the Control group. The sedation score at $30 \mathrm{~min}$ after skin incision, and time to an Aldrete score $>9$ points in the recovery room were similar between the two study groups, which demonstrates that the addition of ketamine did not delay awakening of the patients after the procedures.

Large doses of ketamine $(>2 \mathrm{mg} / \mathrm{kg})$ are frequently associated with unpleasant side effects including hallucinations, dreams, cognitive decline, and emergence confusion. Also, ketamine can cause involuntary muscle movement, potentially limiting its suitability in interventional radiology procedures. However, a small bolus of ketamine $(0.5-1.0 \mathrm{mg} / \mathrm{kg})$ without continuous infusion rarely causes such side effects [25], and there were no patients complaining of hallucinations or confusion in this study. This result is consistent with other studies, where a single bolus of ketamine was used.

In some studies, remifentanil elicited hyperalgesia (increased sensitivity to noxious stimuli) and rapid intolerance, which was associated with the intraoperative opioid dosage. Despite the fact that ketamine was reported to mitigate remifentanil induced hyperalgesia during the postoperative period, we could not assess the presence of hyperalgesia after the procedure. This might be a limitation of this study, although we believe that the degree of pain after PTA is usually mild and the dose of ketamine in this study was not enough to exert a preventive effect on remifentanil induced hyperalgesia.

In conclusion, a small dose of ketamine as an adjunct sedative to the combination of midazolam and remifentanil in patients undergoing PTA produced a better quality of sedation and analgesia than without ketamine, and provided stable respiration without cardiopulmonary deterioration. 


\section{Acknowledgements}

We would like to thank Dr Jae Kwang Shim for his assistance with the study.

This work was supported by the Department of Anaesthesiology and Pain Medicine, Yonsei University College of Medicine, Seoul, Korea

\section{References}

1. Wouters PF, Van Aken H. Magnetic resonance imaging in children: role of the anaesthesiologist. Eur J Anaesthesiol 1997; 14: 236-8.

2. American Society of Anesthesiologists Task Force on Sedation and Analgesia by Non-Anesthesiologists. Practice guidelines for sedation and analgesia by non-anesthesiologists. Anesthesiology 2002; 96: 1004-17.

3. Mandel JE, Hutchinson MD, Marchlinski FE. Remifentanil-midazolam sedation provides hemodynamic stability and comfort during epicardial ablation of ventricular tachycardia. J Cardiovasc Electrophysiol 2011; 22: 464-6.

4. Egan TD, Minto CF, Hermann DJ, Barr J, Muir KT, Shafer SL. Remifentanil versus alfentanil: comparative pharmacokinetics and pharmacodynamics in healthy adult male volunteers. Anesthesiology 1996; 84: 821-33.

5. Westmoreland CL, Hoke JF, Sebel PS, Hug CC Jr, Muir KT. Pharmacokinetics of remifentanil (GI87084B) and its major metabolite (GI90291) in patients undergoing elective inpatient surgery. Anesthesiology 1993; 79: 893-903.

6. Arrowsmith JB, Gerstman BB, Fleischer DE, Benjamin SB. Results from the American Society for Gastrointestinal Endoscopy/U.S. Food and Drug Administration collaborative study on complication rates and drug use during gastrointestinal endoscopy. Gastrointest Endosc 1991; 37: 421-7.

7. Martin ML, Lennox PH. Sedation and analgesia in the interventional radiology department. J Vasc Interv Radiol 2003; 14: 1119-28.

8. Morton NS. Ketamine for procedural sedation and analgesia in pediatric emergency medicine: a UK perspective. Paediatr Anaesth 2008; 18: 25-9.

9. Javery KB, Ussery TW, Steger HG, Colclough GW. Comparison of morphine and morphine with ketamine for postoperative analgesia. Can J Anaesth 1996; 43: 212-5.

10. Bonfreschi V, Giuliani E, Malagnino FC, Navi A, Coppi G, Silingardi $\mathrm{R}$, et al. Analgesia during abdominal aortic aneurysm endovascular repair: remifentanil vs. fentanyl-midazolam--a randomized controlled trial. Eur J Anaesthesiol 2009; 26: 782-7.

11. Ben-Shlomo I, abd-el-Khalim H, Ezry J, Zohar S, Tverskoy M. Midazolam acts synergistically with fentanyl for induction of anaesthesia. Br J Anaesth 1990; 64: 45-7.

12. Vinik HR, Bradley EL Jr, Kissin I. Midazolam-alfentanil synergism for anesthetic induction in patients. Anesth Analg 1989; 69: 213-7.

13. Kissin I, Vinik HR, Castillo R, Bradley EL Jr. Alfentanil potentiates midazolam-induced unconsciousness in subanalgesic doses. Anesth Analg 1990; 71: 65-9.

14. Short TG, Plummer JL, Chui PT. Hypnotic and anaesthetic interactions between midazolam, propofol and alfentanil. Br J Anaesth 1992; 69: 162-7.

15. Glass PS, Gan TJ, Howell S. A review of the pharmacokinetics and pharmacodynamics of remifentanil. Anesth Analg 1999; 89: S7-14.

16. White PF, Way WL, Trevor AJ. Ketamine--its pharmacology and therapeutic uses. Anesthesiology 1982; 56: 119-36.

17. Green SM, Johnson NE. Ketamine sedation for pediatric procedures: Part 2, Review and implications. Ann Emerg Med 1990; 19: 1033-46.

18. Carlsson M, Carlsson A. Schizophrenia: a subcortical neurotransmitter imbalance syndrome? Schizophr Bull 1990; 16: 425-32.

19. Hong W, Short TG, Hui TW. Hypnotic and anesthetic interactions between ketamine and midazolam in female patients. Anesthesiology 1993; 79: 1227-32.

20. Hadi BA, Al Ramadani R, Daas R, Naylor I, Zelko R. Remifentanil in combination with ketamine versus remifentanil in spinal fusion surgery--a double blind study. Int J Clin Pharmacol Ther 2010; 48: 542-8.

21. Parras Maldonado MT, Garcia Saura PL, Carrasco De Andres D. Ketamine combined with remifentanil for pediatric ear surgery. Rev Esp Anestesiol Reanim 2009; 56: 646-8.

22. Lester L, Braude DA, Niles C, Crandall CS. Low-dose ketamine for analgesia in the ED: a retrospective case series. Am J Emerg Med 2010; 28: 820-7.

23. Avramov MN, Smith I, White PF. Interactions between midazolam and remifentanil during monitored anesthesia care. Anesthesiology 1996; 85: 1283-9.

24. Rudner R, Jalowiecki P, Kawecki P, Gonciarz M, Mularczyk A, Petelenz M. Conscious analgesia/sedation with remifentanil and propofol versus total intravenous anesthesia with fentanyl, midazolam, and propofol for outpatient colonoscopy. Gastrointest Endosc 2003; 57: 657-63.

25. Seigler RS, Avant MG, Gwyn DR, Lynch AL, Golding EM, Blackhurst DW, et al. A comparison of propofol and ketamine/midazolam for intravenous sedation of children. Pediatr Crit Care Med 2001; 2: 20-3. 
$<$ Appendix A> OAA/S Scale

\begin{tabular}{|c|c|c|c|c|}
\hline Response & Speech & Facial expression & Eyes & $\begin{array}{l}\text { Composite } \\
\text { score }\end{array}$ \\
\hline $\begin{array}{l}\text { Responds readily to name spoken in } \\
\text { normal tone }\end{array}$ & Normal & Normal & Clear, no ptosis & 5 \\
\hline $\begin{array}{l}\text { Lethargic response to name spoken in } \\
\text { normal tone }\end{array}$ & $\begin{array}{l}\text { Mild slowing or } \\
\text { thickening }\end{array}$ & Mild relaxation & $\begin{array}{l}\text { Glazed or mild ptosis } \\
\text { (less than half the eye) }\end{array}$ & 4 \\
\hline $\begin{array}{l}\text { Responds only after name is called } \\
\text { loudly or repeatedly }\end{array}$ & $\begin{array}{l}\text { Slurring or prominent } \\
\text { slowing }\end{array}$ & $\begin{array}{l}\text { Marked relaxation } \\
\text { (slack jaw) }\end{array}$ & $\begin{array}{l}\text { Glazed and marked ptosis } \\
\text { (half the eye or more) }\end{array}$ & 3 \\
\hline $\begin{array}{l}\text { Responds only after mild prodding or } \\
\text { shaking }\end{array}$ & Few recognizable words & & & 2 \\
\hline $\begin{array}{l}\text { Does not respond to mild prodding or } \\
\text { shaking }\end{array}$ & & & & 1 \\
\hline Does not respond to noxious stimulus & & & & 0 \\
\hline
\end{tabular}

OAA/S: Observer's Assessment of Alertness/Sedation. 